PROCEEDINGS OF THE

AMERICAN MATHEMATICAL SOCIETY

Volume 139, Number 9, September 2011, Pages 3195-3202

S 0002-9939(2011)10753-6

Article electronically published on February 3, 2011

\title{
SUMS WITH CONVOLUTIONS OF DIRICHLET CHARACTERS TO CUBE-FREE MODULUS
}

\author{
AHMET MUHTAR GÜLOĞLU
}

(Communicated by Wen-Ching Winnie Li)

\begin{abstract}
We find estimates for short sums of the form $\sum_{n m \leqslant X} \chi_{1}(n) \chi_{2}(m)$, where $\chi_{1}$ and $\chi_{2}$ are non-principal Dirichlet characters to modulus $q$, a cubefree integer, and $X$ can be taken as small as $q^{\frac{1}{2}+\epsilon}$.
\end{abstract}

\section{INTRODUCTION}

1.1. Notation. Let $\chi_{1}, \chi_{2}$ be non-principal Dirichlet characters to moduli $q_{1}>1$ and $q_{2} \geqslant q_{1}$, respectively. The convolution of $\chi_{1}$ and $\chi_{2}$, denoted $\chi_{1} * \chi_{2}$, is defined formally by the relation

$$
L\left(s, \chi_{1}\right) L\left(s, \chi_{2}\right)=\sum_{n=1}^{\infty} \chi_{1}(n) n^{-s} \sum_{n=1}^{\infty} \chi_{2}(n) n^{-s}=\sum_{n=1}^{\infty}\left(\chi_{1} * \chi_{2}\right)(n) n^{-s} ;
$$

thus,

$$
\left(\chi_{1} * \chi_{2}\right)(n)=\sum_{a b=n} \chi_{1}(a) \chi_{2}(b) .
$$

Using the truncated version of Perron's formula together with available estimates for Dirichlet $L$-functions one can show that the summatory function

$$
\mathcal{S}_{\chi_{1} * \chi_{2}}(X):=\sum_{n \leqslant X}\left(\chi_{1} * \chi_{2}\right)(n)
$$

satisfies the bound (see, e.g., the remark following [4, Theorem 4.16])

$$
\mathcal{S}_{\chi_{1} * \chi_{2}}(X) \ll\left(q_{1} q_{2}\right)^{\frac{1}{3}} X^{\frac{1}{3}+\epsilon},
$$

where the implied constant depends only on $\epsilon$. (See 3 for recent results on related estimates as well as estimates of more general arithmetic functions.)

Note that the above estimate is worse than the trivial estimate

$$
\left|\mathcal{S}_{\chi_{1} * \chi_{2}}(X)\right| \leqslant X \log X
$$

unless $X \geqslant\left(q_{1} q_{2}\right)^{\frac{1}{2}+\epsilon}$.

Received by the editors January 12, 2010 and, in revised form, August 21, 2010.

2010 Mathematics Subject Classification. Primary 11L40.

Key words and phrases. Convolution of Dirichlet characters, Burgess bound.

(C)2011 American Mathematical Society Reverts to public domain 28 years from publication 
1.2. Statement of results. In this paper we estimate $\mathcal{S}_{\chi_{1} * \chi_{2}}(X)$ for small values of $X$ in the case of two non-principal Dirichlet characters $\chi_{1}, \chi_{2}$ with a cube-free integer modulus $q>1$. Our main result in this direction is the following:

Theorem 1. Let $q>1$ be a cube-free integer and $\chi_{1}, \chi_{2}$ non-principal Dirichlet characters to modulus $q$. Fix $\epsilon>0$. Then, for any integer $d>1$ and $X \geqslant q^{\frac{1}{2}+\frac{1}{2 d} \text {, }}$

$$
\mathcal{S}_{\chi_{1} * \chi_{2}}(X) \ll_{\epsilon, d} \min \left\{\mathcal{S}_{1}(d, X), \mathcal{S}_{2}(d, X)\right\} \log X,
$$

where

$$
\mathcal{S}_{1}(d, X)=q^{\frac{2 d^{2}+4 d+1}{4 d(d+1)^{2}}+\epsilon} X^{\frac{d}{d+1}}, \quad \mathcal{S}_{2}(d, X)=q^{\frac{2 d^{2}+d-1}{4 d^{3}}+\epsilon} X^{\frac{2 d^{2}-2 d+1}{2 d^{2}}} .
$$

Theorem 1 provides a non-trivial bound if $X \gg_{\epsilon, d} q^{\frac{1}{2}+\frac{1}{2 d}+\epsilon}$. For the next result, we introduce two numbers:

$$
E(d)=\frac{1}{2}+\frac{1}{2 d}+\frac{1}{2(d-1)} \quad \text { and } \quad A(d)=\frac{E(d+1)+E(d)}{2} \quad(d>1) .
$$

Proposition 2. Let $q, \chi_{1}, \chi_{2}, \epsilon$ be as in Theorem 1. Then, for any integer $d>1$,

$$
\min \left\{\mathcal{S}_{i}\left(d^{\prime}, X\right): d^{\prime}>1, i=1,2\right\}=\left\{\begin{array}{llr}
\mathcal{S}_{1}(d, X) & \text { if } & q^{E(d+1)} \leqslant X<q^{A(d)}, \\
\mathcal{S}_{2}(d, X) & \text { if } & q^{A(d)} \leqslant X<q^{E(d)}
\end{array}\right.
$$

Note that since $E(d+1)>\frac{1}{2}+\frac{1}{2 d}$, the bound in Theorem 1 istill holds when $q^{E(d+1)} \leqslant X<q^{E(d)}$ for any $d>1$.

For comparison with [1, Corollary 2], we state our result explicitly for $d=2$ and $d=3$, which follows by combining Theorems 11 and 2 ,

Corollary 3. Let $q, \chi_{1}, \chi_{2}, \epsilon$ be as in Theorem 1, Then,

$$
\mathcal{S}_{\chi_{1} * \chi_{2}}(X) \ll \log X\left\{\begin{array}{lll}
q^{\frac{31}{192}}+\epsilon X^{\frac{3}{4}} & \text { if } & X \in\left[q^{\frac{19}{24}}, q^{\frac{41}{48}}\right), \\
q^{\frac{5}{27}}+\epsilon X^{\frac{13}{18}} & \text { if } & X \in\left[q^{\frac{41}{48}}, q^{\frac{11}{12}}\right), \\
q^{\frac{17}{72}+\epsilon} X^{\frac{2}{3}} & \text { if } & X \in\left[q^{\frac{11}{12}}, q^{\frac{13}{12}}\right), \\
q^{\frac{9}{32}+\epsilon} X^{\frac{5}{8}} & \text { if } & X \in\left[q^{\frac{13}{12}}, q^{\frac{5}{4}}\right) .
\end{array}\right.
$$

We remark that our method follows mainly that of [1] and consists of dissecting $\mathcal{S}_{\chi_{1 * \chi_{2}}}(X)$ (see section 2.2) and then applying Burgess' bound (see Lemma 4).

1.3. Previous work. An analogue of the sum $\mathcal{S}_{\chi_{1} * \chi_{2}}(X)$ has been previously estimated by Moshchevitin (see the proof of [5. Theorem 5]) in the special case that $\chi_{2}=\bar{\chi}_{1}$ and $q_{1}=q_{2}=p$ is a prime number and has been shown to be important for some problems on continued fractions of rational numbers. This sum also makes its appearance in a paper by Moshchevitin and Ushanov [6], where they generalize a theorem by Larcher on good lattice points and multiplicative subgroups modulo a prime.

More recently, Banks and Shparlinski [1] have estimated $\mathcal{S}_{\chi_{1} * \chi_{2}}(X)$ for primitive Dirichlet characters $\chi_{1}, \chi_{2}$ of conductors $q_{1}>1$, and $q_{2} \geqslant q_{1}$, respectively. Their result improves and generalizes the bounds given in [5] and [6] and holds for $X \geqslant q_{2}^{\frac{2}{3}}$ with $\log X=q_{2}^{o(1)}$. Assuming in our case that the modulus $q$ of the characters is cube-free allows us to make full use of Burgess' bound. In this way, we extend the range of $X$ down to $q^{\frac{1}{2}+\epsilon}$ and achieve a slight improvement (see Corollary 3) over those in [1, Corollary 2]. 
1.4. Related problems. One can consider estimating $\mathcal{S}_{\chi_{1} * \chi_{2}}(X)$ in the case of two characters to distinct moduli, both of which are cube-free. Another direction would be to consider the convolution of a number of Dirichlet characters, namely, to estimate

$$
\sum_{a_{1} \cdots a_{k} \leqslant X} \chi_{1}\left(a_{1}\right) \cdots \chi_{k}\left(a_{k}\right),
$$

where the $\chi_{i}$ are characters to moduli $q_{i}>1$. This is the summatory function associated with the product

$$
L\left(s, \chi_{1}\right) \cdots L\left(s, \chi_{k}\right) .
$$

Note that when the characters are the same, say $\chi$, we have

$$
\mathcal{S}_{\chi * \chi}(X)=\sum_{n \leqslant X} \tau(n) \chi(n),
$$

where $\tau(n)$ is the number of positive divisors of $n$. Related sums of the form

$$
\sum_{n \leqslant X} \tau(n) \chi(n+a) \quad(a \in \mathbb{Z} ; \operatorname{gcd}(a ; q)=1)
$$

have also been studied but are generally approached using different methods.

Using our method one can also estimate the summatory function associated with the more general product

$$
\sum_{n} f(n) n^{-s} \sum_{n} g(n) n^{-s}=\sum_{n}(f * g)(n) n^{-s},
$$

where $f$ and $g$ are two arithmetic functions, as long as one can estimate, for small values of $X$, the sums

$$
\sum_{n \leqslant X} f(n) \quad \text { and } \quad \sum_{n \leqslant X} g(n) .
$$

\section{Proof of Theorem 1 and Proposition 2}

2.1. Preliminaries. The following result, due to D. A. Burgess [2], plays a central role in our work:

Lemma 4. Let $q>1$ be a cube-free integer, $\rho \geqslant 1$ a fixed integer, and $\epsilon>0$ a fixed real number. If $\chi$ is a non-principal Dirichlet character to modulus $q$, then for any pair of integers $M$ and $N>0$,

$$
\sum_{M \leqslant n \leqslant M+N} \chi(n) \ll N^{1-\frac{1}{\rho}} q^{\frac{\rho+1}{4 \rho^{2}}+\epsilon},
$$

where the implied constant depends on $\epsilon$ and $\rho$.

Burgess' bound holds in general for any integer $q>1$, in which case $1 \leqslant \rho \leqslant 3$. This bound is useful, for a fixed $\rho \geqslant 1$, when $N \gg q^{\frac{1}{4}+\frac{1}{4 \rho}+\epsilon}$. In case $q$ is prime, one can prove (see, e.g., what follows [4, Theorem 12.6]) a slightly stronger bound, namely, that

$$
\left|\sum_{M \leqslant n \leqslant M+N} \chi(n)\right| \leqslant 30 N^{1-\frac{1}{\rho}} q^{\frac{\rho+1}{4 \rho^{2}}}(\log q)^{\frac{1}{\rho}} .
$$


2.2. Hyperbola method. We write $\mathcal{S}_{\chi_{1} * \chi_{2}}(X)$ as $S_{1}+S_{2}-S_{3}$, where

$$
S_{1}=\sum_{\substack{n m \leqslant \sqrt{X} \\ n \leqslant \sqrt{X}}} \chi_{1}(n) \chi_{2}(m), \quad S_{2}=\sum_{\substack{n m \leqslant \sqrt{X} \\ m \leqslant \sqrt{X}}} \chi_{1}(n) \chi_{2}(m),
$$

and

$$
S_{3}=\sum_{n \leqslant \sqrt{X}} \chi_{1}(n) \sum_{m \leqslant \sqrt{X}} \chi_{2}(m) .
$$

From now on we shall assume that $\epsilon>0$ is fixed. Using (2) with $\rho=R>1$ we see that

$$
S_{3} \ll E_{R}(\epsilon):=X^{1-\frac{1}{R}} q^{\frac{R+1}{2 R^{2}}+\epsilon} .
$$

2.3. Bounding the sums $S_{1}$ and $S_{2}$. Due to the symmetry and the fact that the bound in (2) depends only on the conductor of the character and not on the character itself, it is enough to estimate $S_{1}$.

Following [1] we introduce two parameters $\theta \in(0,1 / 2)$ and $\gamma \in(1 / 2,1]$, and write $S_{1}$ as $S_{11}+S_{12}$, where

$$
S_{11}=\sum_{\substack{n m \leqslant X \\ n \leqslant \gamma X^{\theta}}} \chi_{1}(n) \chi_{2}(m), \quad S_{12}=\sum_{\substack{n m \leqslant X \\ \gamma X^{\theta}<n \leqslant \sqrt{X}}} \chi_{1}(n) \chi_{2}(m) .
$$

Applying (2) to $S_{11}$ with $\rho=R-1$ we obtain

$$
\left|S_{11}\right| \ll X^{1+\frac{\theta-1}{R-1}} q^{\frac{R}{4(R-1)^{2}}+\epsilon} .
$$

For $R>2$, we choose $\theta=\frac{1}{R}$ and deduce that

$$
\left|S_{11}\right| \ll X^{1-\frac{1}{R}} q^{\frac{R}{4(R-1)^{2}}+\epsilon} \leqslant E_{R}(\epsilon),
$$

since

$$
\frac{R}{4(R-1)^{2}}<\frac{R+1}{2 R^{2}} .
$$

For $R=2$, we choose $\theta=\frac{1}{3}$, obtaining

$$
\left|S_{11}\right| \ll X^{\frac{1}{3}} q^{\frac{1}{2}+\epsilon} \leqslant E_{2}(\epsilon),
$$

whenever $X \geqslant q^{\frac{3}{4}}$.

\subsection{Estimating the sum $S_{12}$. Fix a real number $\lambda$ such that}

$$
3 X^{-\theta}<\lambda \leqslant 1 \text {. }
$$

Let $I$ be the positive integer determined by the relation

$$
(1+\lambda)^{I} \geqslant X^{1 / 2-\theta}>(1+\lambda)^{I-1} .
$$

It immediately follows from this definition that for $X>e^{\frac{\lambda}{2 \theta}}$,

$$
I<1+\frac{\left(\frac{1}{2}-\theta\right) \log X}{\log (1+\lambda)}<\frac{2\left(\frac{1}{2}-\theta\right) \log X+\lambda}{\lambda}<\lambda^{-1} \log X .
$$

If we choose $\gamma=X^{\frac{1}{2}-\theta}(1+\lambda)^{-I}$ we see that

$$
\frac{1}{2} \leqslant(1+\lambda)^{-1}<\gamma \leqslant 1,
$$

that is, $\gamma \in\left(\frac{1}{2}, 1\right]$, as needed. 
Finally, we put $Z_{0}=\gamma X^{\theta}$ and $Z_{i}=Z_{i-1}(1+\lambda)$ for $i=1, \ldots, I$. Notice that $Z_{I}=\sqrt{X}$.

We now rewrite $S_{12}$ as $S_{12}^{\prime}+S_{12}^{\prime \prime}$, where

$$
\begin{aligned}
& S_{12}^{\prime}=\sum_{i=1}^{I} \sum_{Z_{i-1}<n \leqslant Z_{i}} \chi_{1}(n) \sum_{m \leqslant \frac{X}{Z_{i}}} \chi_{2}(m), \\
& S_{12}^{\prime \prime}=\sum_{i=1}^{I} \sum_{Z_{i-1}<n \leqslant Z_{i}} \chi_{1}(n) \sum_{\frac{X}{Z_{i}}<m \leqslant \frac{X}{n}} \chi_{2}(m) .
\end{aligned}
$$

Note that $Z_{i}-Z_{i-1}=\lambda Z_{i-1} \geqslant \lambda Z_{0}>1$. Since

$$
\frac{X}{n}-\frac{X}{Z_{i}}<\frac{X}{Z_{i-1}}-\frac{X}{Z_{i}}<\frac{X \lambda}{Z_{i-1}} \quad(i=1, \ldots, I),
$$

and $X \lambda / Z_{i-1}>X^{\frac{1}{2}-\theta}$, it follows by (9) and (2) applied with $\rho=s$ that

$$
\begin{aligned}
\left|S_{12}^{\prime \prime}\right| & \ll \sum_{i=1}^{I} \lambda Z_{i-1}\left(\frac{X \lambda}{Z_{i-1}}\right)^{1-\frac{1}{s}} q^{\frac{s+1}{4 s^{2}}+\epsilon} \\
& \leqslant q^{\frac{s+1}{4 s^{2}}+\epsilon} \lambda^{1-\frac{1}{s}} X^{1-\frac{1}{2 s}} \log X .
\end{aligned}
$$

As for $S_{12}^{\prime}$, using (2) twice with $\rho=t$ and $\rho=r$ we deduce that

$$
\begin{aligned}
\left|S_{12}^{\prime}\right| & \ll \sum_{i=1}^{I}\left(\lambda Z_{i-1}\right)^{1-\frac{1}{r}} q^{\frac{r+1}{4 r^{2}}+\frac{\epsilon}{2}}\left(\frac{X}{Z_{i-1}}\right)^{1-\frac{1}{t}} q^{\frac{t+1}{4 t^{2}}+\frac{\epsilon}{2}} \\
& \leqslant q^{\frac{r+1}{4 r^{2}}+\frac{t+1}{4 t^{2}}+\epsilon} \lambda^{-\frac{1}{r}} X^{1-\frac{r+t}{2 r t}} \log X .
\end{aligned}
$$

We now choose $\lambda=\lambda(s, r, t)$ in order to balance (10) and (11); that is, we set

$$
\lambda(s, r, t)=X^{f(s, r, t)} q^{g(s, r, t)},
$$

where

$$
\begin{aligned}
& f(s, r, t)=\left(\frac{1}{2 s}-\frac{1}{2 r}-\frac{1}{2 t}\right)\left(1+\frac{1}{r}-\frac{1}{s}\right)^{-1}, \\
& g(s, r, t)=\left(\frac{r+1}{4 r^{2}}+\frac{t+1}{4 t^{2}}-\frac{s+1}{4 s^{2}}\right)\left(1+\frac{1}{r}-\frac{1}{s}\right)^{-1} .
\end{aligned}
$$

Here the parameters $s, r$ and $t$ must be chosen so that (8) is satisfied. Assuming this holds for some triple $(s, r, t)$, we conclude upon combining (10) and (11) that

$$
\left|S_{12}\right| \ll B_{X, q}^{\epsilon}(s, r, t):=X^{F(s, r, t)} q^{G(s, r, t)+\epsilon} \log X,
$$

where

$$
\begin{aligned}
& F(s, r, t)=\frac{s-1}{s} f(s, r, t)+1-\frac{1}{2 s}, \\
& G(s, r, t)=\frac{s-1}{s} g(s, r, t)+\frac{s+1}{4 s^{2}} .
\end{aligned}
$$

From now on we shall omit the subscripts $X$ and $q$, and write $B^{\epsilon}(s, r, t)$ instead of $B_{X, q}^{\epsilon}(s, r, t)$. 
2.5. Proof of Theorem 1, Combining (5), (7) and (13) we conclude that

$$
\begin{aligned}
\left|\mathcal{S}_{\chi_{1} * \chi_{2}}(X)\right| & \leqslant\left|S_{1}\right|+\left|S_{2}\right|+\left|S_{3}\right| \leqslant 2\left(\left|S_{11}\right|+\left|S_{12}\right|\right)+\left|S_{3}\right| \\
& \ll B^{\epsilon}(s, r, t)+E_{R}(\epsilon) \leqslant \max \left\{B^{\epsilon}(s, r, t), E_{R}(\epsilon)\right\},
\end{aligned}
$$

where $(s, r, t)$ is a triple for which (8) holds with $\theta=\frac{1}{R}$ if $R>2$ and with $\theta=\frac{1}{3}$ if $R=2$, in which case we assume that $X \geqslant q^{\frac{3}{4}}$.

Fix an integer $d>1$, and take all parameters $R, s, r, t$ equal to $d$. One can then easily check that for $X \geqslant q^{\frac{1}{2}+\frac{1}{2 d}}$,

$$
E_{d}(\epsilon) \leqslant B^{\epsilon}(d, d, d)=\mathcal{S}_{2}(d, X) \log X,
$$

and that (8) is satisfied with these parameters.

Similarly, if we choose $R, s, r=d+1$ and $t=d$, then for $X \geqslant 1$,

$$
E_{d+1}(\epsilon) \leqslant B^{\epsilon}(d+1, d+1, d)=\mathcal{S}_{1}(d, X) \log X,
$$

and one can easily verify that (8) holds with these parameters as well. This establishes the proof of Theorem 1

2.6. Proof of Proposition 2. We first note that the inequality

$$
\mathcal{S}_{2}(d, X) \leqslant \mathcal{S}_{1}(d, X)
$$

holds if and only if $X \geqslant q^{A(d)}$, while

$$
\mathcal{S}_{1}(d, X) \leqslant \mathcal{S}_{2}(d+1, X)
$$

holds if and only if $X \geqslant q^{E(d+1)}$. This implies that the minimal choice among $S_{i}\left(d^{\prime}, X\right)$ with $i=1,2$ and $d^{\prime}>1$ is $\mathcal{S}_{1}(d, X)$ for $q^{E(d+1)} \leqslant X<q^{A(d)}$, and $\mathcal{S}_{2}(d, X)$ for $q^{A(d)} \leqslant X<q^{E(d)}$. This concludes the proof of Proposition 2

\section{Determining the optimal Bound}

The following result justifies our choice of the triples $(d, d, d)$ and $(d+1, d+1, d)$ in the proof of Theorem 1 among other possible triples $(s, r, t)$ for which $s, r, t \geqslant d$ :

Lemma 5. For any integer $d>1$ and any choice of triples $(s, r, t)$ with $s, r, t \geqslant d$, we have

$$
\begin{aligned}
& B^{\epsilon}(d+1, d+1, d) \leqslant B^{\epsilon}(s, r, t), \quad \text { if } \quad q^{E(d+1)} \leqslant X<q^{A(d)}, \\
& B^{\epsilon}(d, d, d) \leqslant B^{\epsilon}(s, r, t), \quad \text { if } \quad q^{A(d)} \leqslant X<q^{E(d)} .
\end{aligned}
$$

Proof. Note that for triples $(s, r, t)$ and $\left(s^{\prime}, r^{\prime}, t^{\prime}\right)$, the inequality

$$
B^{\epsilon}(s, r, t) \leqslant B^{\epsilon}\left(s^{\prime}, r^{\prime}, t^{\prime}\right)
$$

holds whenever $X \leqslant q^{\mathcal{P}\left(s, r, t ; s^{\prime}, r^{\prime}, t^{\prime}\right)}$, where

$$
\mathcal{P}\left(s, r, t ; s^{\prime}, r^{\prime}, t^{\prime}\right):=\frac{G\left(s^{\prime}, r^{\prime}, t^{\prime}\right)-G(s, r, t)}{F(s, r, t)-F\left(s^{\prime}, r^{\prime}, t^{\prime}\right)},
$$

provided that both the numerator and denominator of (14) are positive. In case the denominator vanishes, we only require that the numerator be non-negative.

We now choose $s^{\prime}=r^{\prime}=d+1$ and $t^{\prime}=d$ and compute (14). With the aid of a computer or otherwise, one can easily check that 
(1) For $s, r \geqslant 0$, not both zero,

$$
\begin{aligned}
& \quad F(d+1+s, d+1+r, d)-F(d+1, d+1, d) \\
& =\frac{(d-1)(r d+s+r s)}{2 d(1+d)\left(1+2 s+r s+2 d+r d+s d+d^{2}\right)}>0, \\
& G(d+1, d+1, d)-G(d+1+s, d+1+r, d) \\
& =\left\{r^{2}\left(d^{3}+d^{2}-2 d-1\right)\left(s+s^{2}+2 d s+d^{2}+d\right)\right. \\
& \quad+s(1+d)\left(d^{4}+3 d^{3}-3 d-1+s\left(d^{3}+d^{2}-2 d-1\right)\right) \\
& +r(2 s+d)(1+d)^{2}\left(d^{3}+2 d^{2}-2 d-1\right) \\
& \left.+r s^{2}\left(d^{4}+4 d^{3}+d^{2}-5 d-2\right)\right\}\left(4 d^{2}(1+Q(d, s, r))\right)^{-1}>0,
\end{aligned}
$$

where $Q(d, s, r)$ is a polynomial with positive coefficients, and

$$
\begin{aligned}
\mathcal{P}(d & +1+s, d+1+r, d ; d+1, d+1, d) \\
& =E(d+1)-\frac{d\left(r^{2}\left(d+d^{2}+s+2 d s\right)+s^{2}\left(1+r+r^{2}+d\right)\right)}{2\left(d^{2}-1\right)(1+r+d)(1+d+s)(r d+s+r s)} \\
& <E(d+1) .
\end{aligned}
$$

(2) For non-negative integers $s, r$ and $t$, and $d^{\prime}=d+1$,

$$
\begin{gathered}
F\left(d^{\prime}+s, d^{\prime}+r, d^{\prime}+t\right)-F\left(d^{\prime}, d^{\prime}, d\right)=\frac{1+P_{1}(d, s, r, t)}{2+Q_{1}(d, s, r, t)}>0, \\
G\left(d^{\prime}, d^{\prime}, d\right)-G\left(d^{\prime}+s, d^{\prime}+r, d^{\prime}+t\right)=\frac{1+P_{2}(d, s, r, t)}{4 d+Q_{2}(d, s, r, t)}>0, \\
\mathcal{P}\left(d^{\prime}+s, d^{\prime}+r, R+t ; d^{\prime}, d^{\prime}, d\right)=E\left(d^{\prime}\right)-\frac{r^{2} d^{2}+s^{2} d+t d+P_{3}(d, s, r, t)}{2 d+Q_{3}(d, s, r, t)},
\end{gathered}
$$

where $P_{i}(d, s, r, t)$ and $Q_{i}(d, s, r, t), i=1,2,3$, are polynomials in $d, s, r, t$ with positive coefficients and $P_{3}(d, 0,0,0)=0$. It follows from the last equation that

$$
\mathcal{P}\left(d^{\prime}+s, d^{\prime}+r, d^{\prime}+t ; d^{\prime}, d^{\prime}, d\right)<E\left(d^{\prime}\right)
$$

unless $r, s$ and $t$ are all zero, in which case we have equality.

(3) For any $d>1$,

$$
\begin{aligned}
& \mathcal{P}(d, d+1, d+1 ; d+1, d+1, d)=E(d+1)-\frac{1}{2 d\left(d^{2}-1\right)}, \\
& \mathcal{P}(d+1, d, d+1 ; d+1, d+1, d)=E(d+1)-\frac{1}{2(d+1)} .
\end{aligned}
$$

(4) For any $X \geqslant 1$,

$$
B^{\epsilon}(d+1, d+1, d)<B^{\epsilon}(d, d, d+1) .
$$


(5) For $X \leqslant q^{A(d)}$, and $d^{\prime}=d+1$, $B^{\epsilon}\left(d^{\prime}, d^{\prime}, d\right) \leqslant \min \left\{B^{\epsilon}\left(d^{\prime}, d, d\right), B^{\epsilon}\left(d, d^{\prime}, d\right), B^{\epsilon}(d, d, d)\right\}$, and for $X \geqslant q^{A(d)}$, $B^{\epsilon}(d, d, d) \leqslant \min \left\{B^{\epsilon}\left(d^{\prime}, d, d\right), B^{\epsilon}\left(d, d^{\prime}, d\right), B^{\epsilon}\left(d, d, d^{\prime}\right), B^{\epsilon}\left(d^{\prime}, d^{\prime}, d\right)\right\}$.

The result follows upon combining all the comparisons above.

\section{ACKNowledgements}

I would like to thank Igor Shparlinski for suggesting the problem and for his remark that allowed for a generalization of the initial result. I would also like to thank William Banks for discussing this problem with me during my visit in Summer 2009. I express my gratitude to the University of Missouri, Columbia, for their hospitality during this visit. Finally, I thank the referee for helpful comments and suggestions which greatly enhanced the exposition of this paper.

\section{REFERENCES}

[1] W. D. Banks, I. Shparlinski, Sums with convolutions of Dirichlet characters, preprint, 2009.

[2] D. A. Burgess, On character sums and L-series. II, Proc. London Math. Soc. (3) 13 (1963), 524-536. MR0148626(26:6133)

[3] J. B. Friedlander and H. Iwaniec, Summation formulae for coefficients of L-functions, Canad. J. Math., 57 (2005), 494-505. MR2134400 (2006d:11095)

[4] H. Iwaniec, E. Kowalski, Analytic number theory, Amer. Math. Soc., Providence, RI, 2004. MR2061214 (2005h:11005)

[5] N. G. Moshchevitin, Sets of the form $\mathcal{A}+\mathcal{B}$ and finite continued fractions, Matem. Sbornik (Transl. as Sbornik: Mathematics) 198 (4) (2007), 95-116 (in Russian). MR 2352362 (2009b:11133)

[6] N. G. Moshchevitin and D. M. Ushanov, On Larcher's theorem concerning good lattice points and multiplicative subgroups modulo p, Unif. Distrib. Theory 5 (1) (2010), 45-52.

Department of Mathematics, Bilkent University, Bilkent, 06800 Ankara, Turkey

E-mail address: guloglua@fen.bilkent.edu.tr 